\title{
A procedure to evaluate the efficiency of surface sterilization methods in culture-independent fungal endophyte studies
}

\author{
R.J. Burgdorf ${ }^{1}$, M.D. Laing ${ }^{2}$, C.D. Morris ${ }^{3}$, S.F. Jamal-Ally ${ }^{1}$ \\ ${ }^{1}$ Department of Microbiology, University of KwaZulu-Natal, Pietermaritzburg, South Africa. \\ ${ }^{2}$ Department of Plant Pathology, University of KwaZulu-Natal, Pietermaritzburg, South Africa. \\ ${ }^{3}$ Agricultural Research Council, c/o School of Life Sciences, University of KwaZulu-Natal, \\ Pietermaritzburg, South Africa.
}

Submitted: June 24, 2013; Approved: March 14, 2014.

\begin{abstract}
Extraneous DNA interferes with PCR studies of endophytic fungi. A procedure was developed with which to evaluate the removal of extraneous DNA. Wheat (Triticum aestivum) leaves were sprayed with Saccharomyces cerevisiae and then subjected to physical and chemical surface treatments. The fungal ITS1 products were amplified from whole tissue DNA extractions. ANOVA was performed on the DNA bands representing $S$. cerevisiae on the agarose gel. Band profile comparisons using permutational multivariate ANOVA (PERMANOVA) and non-metric multidimensional scaling (NMDS) were performed on DGGE gel data, and band numbers were compared between treatments. Leaf surfaces were viewed under variable pressure scanning electron microscopy (VPSEM). Yeast band analysis of the agarose gel showed that there was no significant difference in the mean band DNA quantity after physical and chemical treatments, but they both differed significantly $(\mathrm{p}<0.05)$ from the untreated control. PERMANOVA revealed a significant difference between all treatments $(\mathrm{p}<0.05)$. The mean similarity matrix showed that the physical treatment results were more reproducible than those from the chemical treatment results. The NMDS showed that the physical treatment was the most consistent. VPSEM indicated that the physical treatment was the most effective treatment to remove surface microbes and debris. The use of molecular and microscopy methods for the post-treatment detection of yeast inoculated onto wheat leaf surfaces demonstrated the effectiveness of the surface treatment employed, and this can assist researchers in optimizing their surface sterilization techniques in DNA-based fungal endophyte studies.
\end{abstract}

Key words: endophyte, fungi, DNA, surface sterilization.

\section{Introduction}

Endophytes are microorganisms that live within the tissues of plants without causing damage (Backman and Sikora, 2008). Research which focuses on these organisms must exclude those microbes found on the surfaces of host plants including the lipophilic waxy plant cuticle surface, which is colonized by various fungi and bacteria (Müller and Riederer, 2005). More micro-organisms are to be found on the aerial surfaces of a plant than within plant tissues (Lindow and Brandl, 2003), which emphasizes the impor- tance of extraneous DNA removal as it can affect the conclusions of culture-independent endophyte studies.

The removal of these plant surface microbes and their DNA is particularly important when using a PCR-based approach to investigate endophytes. The use of standard surface sterilization techniques employed in culture-dependent research may not guarantee the complete removal of surface organisms (Anand et al., 2006; Manter et al., 2010), so they cannot guarantee the removal of the DNA belonging to these organisms either, in addition to any ambient DNA that may be present. While surfaces are often tested for microbial sterility by plating the post-treated surface

Send correspondence to R.J. Burgdorf. Department of Microbiology, University of KwaZulu-Natal, Pietermaritzburg, 3201 South Africa. E-mail: burgdorfr@ukzn.ac.za. 
onto a nutrient agar (Sessitsch et al., 2002), Guo (2010) warned that some surface sterilization methods may not sufficiently denature epiphytic DNA in molecular studies of endophytes.

The efficiency of surface sterilization of plants in culture-based endophyte studies (Schulz et al., 1993), the removal of surface fungal DNA from insects (Meyer and Hoy, 2008), as well as DNA removal from the surface of bones and teeth (Kemp and Smith, 2005) has been evaluated. However, the efficiency of surface DNA removal techniques from plant tissues has not been established definitively.

The aim of this investigation was to develop a procedure in which the efficacy of commonly adopted surface sterilization approaches to removing non-endophytic DNA could be evaluated. Saccharomyces cerevisiae Meyen ex E.C. Hansen was used as a test organism on winter wheat (Triticum aestivum L.), and statistical analysis methods were employed to draw conclusions from the outcomes of surface treatments determined by the presence of PCRdetected $S$. cerevisiae and other microbial DNA. Support for these conclusions was provided by electron microscopy.

\section{Materials and Methods}

\section{Wheat cultivation and sample preparation}

Wheat (Triticum aestivum L. cv Duzi) was planted in $300 \mathrm{~mm}$ pots at a plant density equivalent to $47 \mathrm{~kg}$ of seed per hectare, on the $11^{\text {th }}$ of July 2010 in Pietermaritzburg, KwaZulu-Natal, South Africa. Plants were grown outside under $10 \%$ shade, in composted pine bark, with drip irrigation providing 4:1:3 NPK (N at $200 \mathrm{ppm}$ ) for 10 min 3 times per day. Mean rainfall, mean high and mean low temperatures over the growth period were $0.1 \mathrm{~mm}, 24.3^{\circ} \mathrm{C}$ and $10.5^{\circ} \mathrm{C}$ respectively.

Four replicates pots were used for the treatments, and for the positive control and the negative control $(n=16)$, arranged in a completely randomized design. On the $22^{\text {nd }}$ of September 2010, at growth stage 60 (Tottman, 1987), leaves in each of the four replicates of the two treatments and the positive control pots were inoculated with $100 \mathrm{~mL}$ of yeast broth inoculum per pot. Inoculum was sprayed using a Fragram (Carrara, Queensland, Australia) 1.5 L pressure sprayer, which delivered the broth culture in a fine mist.

The total volume of $1.2 \mathrm{~L}$ of inoculum consisted of malt extract broth (MEB), made from $30 \mathrm{~g} \mathrm{~L}^{-1}$ malt extract (Merck, Darmstadt, Germany) and $2.5 \mathrm{~g} \mathrm{~L}^{-1}$ yeast extract (Merck) in distilled water. This was autoclaved for $15 \mathrm{~min}$ at $121{ }^{\circ} \mathrm{C}$, cooled to room temperature and inoculated with $1.7 \mathrm{~g} \mathrm{~L}^{-1}$ of dried $S$. cerevisiae granules (instant baking yeast from NCP, Johannesburg, South Africa). The broth was incubated in a Model TU-453 shaking incubator
(MRC, Holon, Israel) at $25^{\circ} \mathrm{C}$ for $18 \mathrm{~h}$, reaching a viable cell concentration of $1.02 \times 10^{8} \mathrm{cfu} \mathrm{mL}^{-1}$.

\section{Sample collection and surface treatments}

After 72 h, during which no rain fell and mean high and mean low temperatures were $24.4{ }^{\circ} \mathrm{C}$ and $12.7^{\circ} \mathrm{C}$ respectively, leaves were harvested. Four $0.1 \mathrm{~g}$ replicates of sprayed leaves were subjected to two treatments (A and B). The experimental controls comprised a positive control (Y), with four replicates of wheat leaf tissue that were sprayed with yeast broth, but not surface treated, and a negative control $(\mathrm{N})$, which consisted of four replicates of wheat leaf tissue that were not sprayed with yeast broth and were not surface treated in any way.

The Physical Treatment (A) was a modification of the method used by Sessitsch et al. (2002). Leaf samples (0.1 g) were placed in McCartney bottles with $20 \mathrm{~mL}$ of a $0.01 \%$ water solution of Tween 20 (Merck) and sonicated for $5 \mathrm{~min}$ in a Biosonic sonication bath (Colténe/Whaledent, Altstätten, Switzerland). Leaf samples were rinsed once with tap water and once with sterile distilled water. Samples were placed in a $2 \mathrm{~mL}$ microtube with $1.5 \mathrm{~mL} 0.9 \%$ $\mathrm{NaCl}$ solution and $0.3 \mathrm{~g}$ of $0.1 \mathrm{~mL}$ acid washed beads (Sigma-Aldrich, St. Louis, MO, USA). The tubes were vortexed on a Disruptor Genie Vortex (Scientific Industries, Inc., Bohemia, NY, USA) for $20 \mathrm{~min}$. Samples were rinsed three times in $1 \mathrm{~mL}$ of sterile ultra-pure water and then stored individually in plastic bags and frozen at $-80{ }^{\circ} \mathrm{C}$ before further processing.

The Chemical Treatment (B) was according to the method described by Arnold et al. (2007). Leaf samples $(0.1 \mathrm{~g})$ were immersed in a $95 \%$ ethanol solution for $5 \mathrm{~s}$, followed by $2 \mathrm{~min}$ in a $\mathrm{NaOCl}$ solution $\left(0.5 \%\right.$ free $\left.\mathrm{Cl}_{2}\right)$ and finally $2 \mathrm{~min}$ in $70 \%$ ethanol. After that the samples were dried in a laminar flow hood. Samples were stored individually in plastic bags and frozen at $-80^{\circ} \mathrm{C}$ before further processing.

\section{DNA extraction and amplification}

Four replicate leaf samples $(0.1 \mathrm{~g})$ for each treatment and controls were ground in liquid nitrogen with the addition of $0.1 \mathrm{~mm}$ sterile acid-washed beads (Sigma-Aldrich) and the DNA was extracted using the CTAB protocol for the Nucleospin Plant II Genomic DNA extraction kit (Macherey-Nagel, Düren, Germany). S. cerevisiae genomic DNA was extracted from yeast cells pelleted from $1 \mathrm{~mL}$ of the same broth used for inoculation, according to the same method as for the plant tissue. Working solutions of all genomic DNA were made up to a final concentration of $10 \mathrm{ng} \mu \mathrm{L}^{-1}$ using nuclease free water (Promega, Madison, WI, USA).

All PCR reactions were performed using the KAPA2G Fast HotStart ReadyMix Kit (Kapa Biosystems, Woburn, MA, USA) and universal fungal primers ITS1FGC forward primer (5'-CGC CCG CCG CGC CCC GCG 
CCC GGC CCG CCG CCC CTT GGT CAT TTA GAG GAA GTA A-3') and the ITS2 reverse primer (5'-TTY GCT GYG TTC TTC ATC G-3') (Wakelin et al., 2007). The dNTP's were at a $0.2 \mathrm{mM}$ concentration, $\mathrm{MgCl}_{2}$ at $1.5 \mathrm{mM}$, and forward and reverse primers at a final concentration of $800 \mathrm{nM}$ each. The final reaction volume was $20 \mu \mathrm{L}$. A sample of $8 \mathrm{ng}$ of genomic DNA template was added to each reaction tube. PCR was performed on a G-storm Goldblock Thermal Cycler (Syngene, Cambridge, United Kingdom). The PCR program consisted of a $2 \mathrm{~min}$ denaturation at $95{ }^{\circ} \mathrm{C}$ followed by 35 cycles at $95{ }^{\circ} \mathrm{C}$ for $15 \mathrm{~s}, 55^{\circ} \mathrm{C}$ for $30 \mathrm{~s}$ and $72^{\circ} \mathrm{C}$ for $10 \mathrm{~s}$. This was followed by a final elongation at $72{ }^{\circ} \mathrm{C}$ for $30 \mathrm{~s}$.

PCR products and a GeneRuler ${ }^{\mathrm{TM}} 100$ bp ladder marker (Thermo Fisher Scientific, Waltham, MA, USA) were run on 1.5\% Seakem LE Agarose (Lonza, Basel, Switzerland) gel containing SYBR ${ }^{\circledR}$ Safe nucleic acid stain (Invitrogen, Carlsbad, California) at $5 \mathrm{~V} \mathrm{~cm}^{-1}$ for $1 \mathrm{~h}$ in a $1 \mathrm{x}$ TBE buffer. The gel was visualized and images captured with the GeneSnap Software on the I-chemi G-Box (Syngene) and analyzed using the GeneTools software (Syngene).

\section{Agarose gel analysis}

The bands corresponding to the band position of the S. cerevisiae amplicons in each sample were quantified relative to the molecular weight marker band representing a molecular weight of $500 \mathrm{bp}$ and a quantity of $115 \mathrm{ng}$ of DNA, in accordance with the manufacturer's instructions (Thermo Fisher Scientific). The quantity of DNA of the band corresponding to the position of the pure S. cerevisiae amplicon was therefore used as a measure of the efficiency of the surface treatment. Means of band DNA quantity for the four replicates for Treatments A, B and the positive control (Y) were compared with a one-way analysis of variance (ANOVA), in Genstat (Payne et al., 2011).

\section{Denaturing gradient gel electrophoresis (DGGE) gel analysis}

PCR products were run on a DGGE gel. The gel was run on a Bio-Rad Dcode ${ }^{\mathrm{TM}}$ Universal Mutation Detection System (Bio-Rad, Hercules, California, USA). A sample of $20 \mu \mathrm{L}$ of PCR product per well was run on a $6 \%$ Acrylamide/Bisacrylamide (40\%, 19:1, Sigma-Aldrich) gel with a 27 to $44 \%$ denaturation gradient, for $16.5 \mathrm{~h}$ at $100 \mathrm{~V}$ in a $60^{\circ} \mathrm{C} 1 \times$ TAE buffer. The gel was stained in a $1 \times$ SYBR ${ }^{\circledR}$ Gold nucleic acid stain (Invitrogen) for $40 \mathrm{~min}$ and the image captured on the I-chemi G-box Gel Documentation system (Syngene). A band presence matrix was produced and band pixel intensity was determined using Quantity One Gel Analysis Software (Bio-Rad).

Contour maps are able to display three-dimensional information in two dimensions: in this case, DGGE band positions and intensity. Band pixel intensity, as a percentage of the pixel intensity value of the brightest band on the gel, was square-root transformed and plotted on a contour map, along with band position, using the gplots library (Warnes et al., 2013) in R (R Core Team, 2013) and edited in Microsoft Windows Paint (2010).

Permutational Multivariate ANOVA (PERMANOVA) implements a flexible non-parametric distancebased analogue of analysis of variance for multivariate data that provides a distribution-free means of testing differences between treatments in their multivariate profile (Anderson, 2001). This was used to test for differences in band composition among treatments.

Non-metric multidimensional scaling (NMDS) provides a robust method of visualizing differences in composition within and between treatments (McCune et al., 2002). NMDS projects multivariate distances among samples in low dimensional space so that distances between projected sample points best approximate their original multivariate differences: sites close in the graph are most similar in their overall composition and sites located at opposite ends of the plotted dimensions have a distinct multivariate profile. This was applied to replicate samples within and among treatments. The software package Primer (v. 6) (Clarke and Gorley, 2006) was used for both multivariate analyses.

An unpaired Student's t-test comparing band numbers (excluding the yeast bands) for Treatments $\mathrm{A}$ and $\mathrm{B}$, was performed in Microsoft Excel (2010).

\section{Variable pressure scanning electron microscopy (VPSEM)}

A leaf segment was taken for Treatments A, B and the two controls, and viewed under a Zeiss Evo LF-15 Variable Pressure Scanning Electron Microscope (Zeiss, Oberkochen, Germany). Samples did not require treatment before viewing (Stokes, 2008). Sample surfaces were observed at a working distance from $7 \mathrm{~mm}$ to $7.5 \mathrm{~mm}$, $15 \mathrm{kGV}$, between $2000 \mathrm{x}$ and $4350 \mathrm{x}$ magnifications and 0.89 to 0.9 Torr pressure. Representative micrographs were captured to demonstrate differences in surface characteristics due to the treatments.

\section{Results}

\section{Agarose gel analysis}

In Figure 1 the presence of $S$. cerevisiae from the original inoculum was confirmed by a band corresponding to that of the amplified ITS1 region from pure $S$. cerevisiae genomic DNA that was used as the positive PCR control. There were no native endophytes or epiphytes that shared the same sized ITS1 fragment as $S$. cerevisiae in the noninoculated control samples. There were visible differences in intensity of the band representing the $S$. cerevisiae amplicon in Treatments A, B and the positive control.

The raw data of individual band DNA quantities were transformed to the square root of the measured values. 


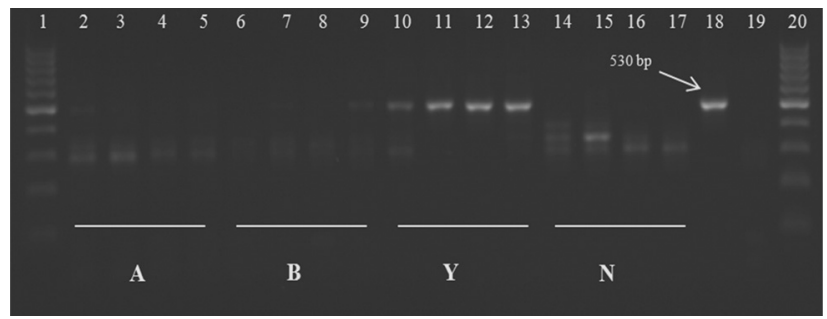

Figure 1 - PCR of all treatments. Lanes 1 and 20, 100 bp molecular weight marker (Thermo Fisher Scientific); Lanes 2 to 5, Treatment A (physical); Lanes 6 to 9, Treatment B (chemical); Lanes 10 to 13, Control Y; Lanes 14 to 17 , Control N; Lane 18, positive PCR control from pure yeast DNA; Lane 19, template-free control. The $S$. cerevisiae band is absent in the negative control samples and the treatments and positive controls show differing intensities of the band corresponding to the $530 \mathrm{bp}$ pure $S$. cerevisiae band.

ANOVA of the transformed data (Table 1) showed no significant difference between Treatments A and B, although they differed significantly from the positive control (Y).

\section{DGGE gel analysis}

The contour map (Figure 2) of DGGE gel data showed differences in the banding patterns, as a result of surface treatments, and in the controls. Region $\mathrm{P}$ accommodated the two bands for the yeast ITS1 amplicon (S) in the positive control samples $(\mathrm{Y})$, which were present, to some extent in surface treated samples (A and B), but absent in the samples that were not inoculated. Region $Q$ consisted of the bands which were greatly reduced by the surface treatments. Region $\mathrm{R}$ was populated by bands present in all samples to a varying degree.

The PERMANOVA analysis (Pseudo-F $=5.7151)$ indicated a significant difference $(\mathrm{p}=0.0001)$ in band composition amongst treatments. Pair-wise tests established that all treatments differed from one another $(p<0.05)$ in their banding patterns.

Mean similarity values (Table 2) provided a measure of the magnitude of the differences in band composition between and within treatments.

The NMDS plot (Figure 3) illustrated the variation within and among treatments quantified in Table 2. The proximity of chemically and physically treated samples in the plot confirmed their similarity, though Physical Treatment (A) samples were less dispersed in the plot, than samples subject to Chemical Treatment (B). Both were dissimilar in composition to the samples from the controls. NMDS including peak intensity data for each band showed a similar pattern of treatment effects on DGGE band profiles (result not shown).

The Student's t-test showed mean band numbers did not differ significantly $(\mathrm{p}>0.05)$ between Treatments A and $\mathrm{B}$.

\section{VPSEM}

The VPSEM micrographs of uninoculated leaf surfaces of the Negative Control (N) (Figure 4A) indicated extraneous microbial hyphae and debris on the leaf surface, while abundant yeast cells were present on the surfaces of the Positive Control (inoculated and untreated leaf surfaces) (Figure 4B). Micrographs of leaf surfaces exposed to the two treatments indicated the degree of removal of particulates adhering to the surface was more effective with physical abrasion (Figure 4C) than with chemical treatment (Figure 4D).

\section{Discussion}

The yeast $S$. cerevisiae was chosen as an epiphytic indicator of surface treatment efficiency because it does not appear to have been isolated in previous culture-based endophyte studies of wheat (Crous et al., 1995; Larran et al., 2002). In addition, PCR amplification revealed that the ITS1 region amplicon derived from $S$. cerevisiae was discernible from native fungal DNA on or within the leaf and it was absent from those leaves which were not sprayed with yeast (Figure 1). This demonstrated that $S$. cerevisiae can serve as a useful inoculant in determining the removal of epiphytic fungal DNA from the leaf.

The goal of a surface treatment method is to remove as much DNA from the surface of the plant while doing minimal damage to endophytic fungal DNA; the relative efficiency of a surface treatment could be inferred from the degree to which yeast DNA could still be detected in DNA extracts from leaf tissue after the treatments.

Table 1 - Results of ANOVA analysis of yeast ITS1 fragment band intensity.

\begin{tabular}{lccc}
\hline & Mean \pm (standard error) & Square-root transformed mean \pm (standard error) & CV\% \\
\hline Physical treatment (A) & $1.37 \pm 0.732$ & $1.00 \pm 0.357 \mathrm{a}$ & 72.39 \\
Chemical treatment (B) & $3.43 \pm 2.934$ & $1.31 \pm 0.755 \mathrm{a}$ & 115.34 \\
No treatment with Yeast (Y) & $60.29 \pm 33.331$ & $7.62 \pm 0.870 \mathrm{~b}$ & 22.84 \\
F-value 2.9 & & 28.787 & $<0.001$ \\
P-value & & 2.227 \\
LSD $(\mathrm{p}>0.05)$ & & & \\
\hline
\end{tabular}

Means with the same letters are not significantly different $(p>0.05)$. CV percentages were of transformed data. 


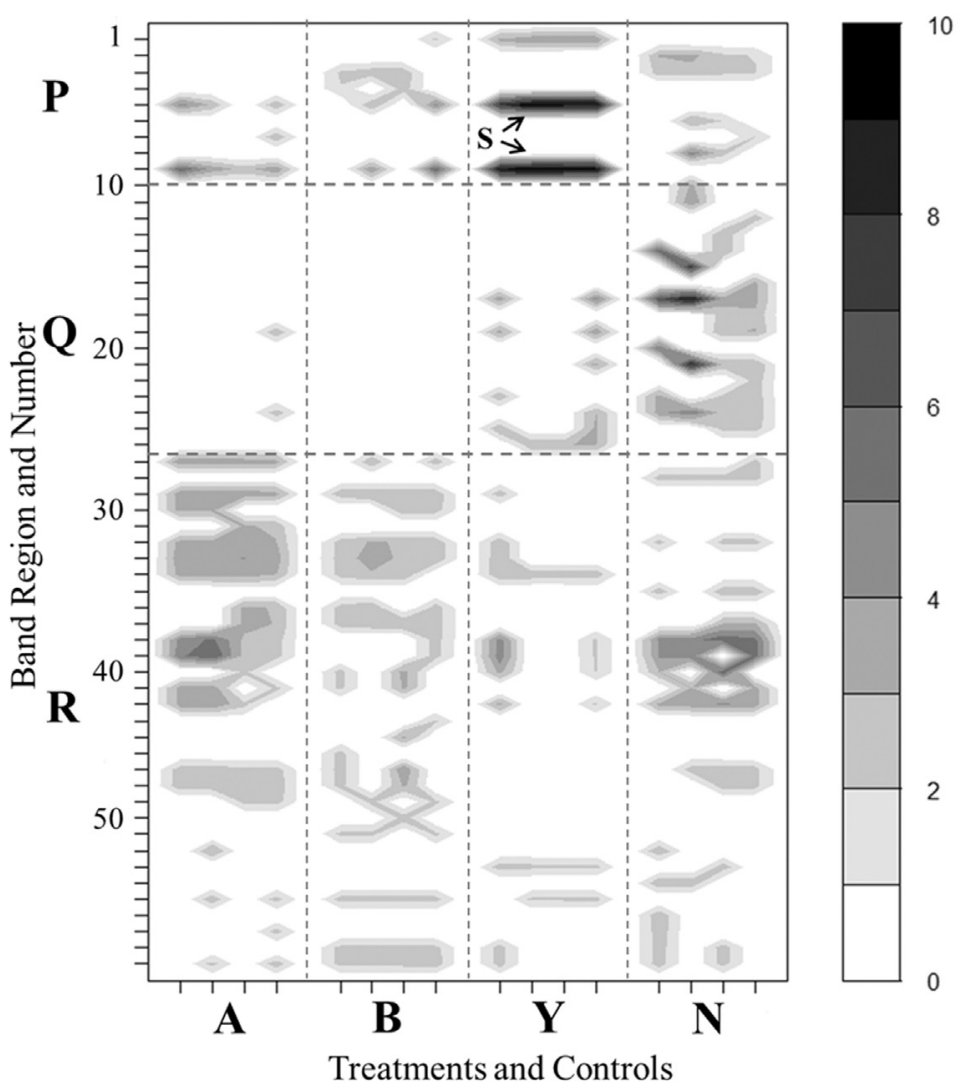

Figure 2 - Contour map indicating band position and intensities from the DGGE gel data. This shows different regions (P, Q and R) with varying effects of surface and control treatments in the columns A, B, Y and N. The yeast ITS1 fragment is represented by two bands (S) in Region P. The key indicates the increasing intensity of the band with increasing darkness, corresponding to the square root of the percentage of maximum band intensity.

Table 2 - Mean similarity (\%) between/within groups from pairwise PERMANOVA analysis.

\begin{tabular}{lcccc}
\hline Treatment & Physical & Chemical & Yeast & None \\
\hline A- Physical & 59.10 & & & \\
B- Chemical & 38.72 & 48.46 & & \\
Y- Yeast & 23.41 & 18.48 & 48.67 & \\
N- Negative & 19.40 & 13.86 & 12.73 & 39.32 \\
\hline
\end{tabular}

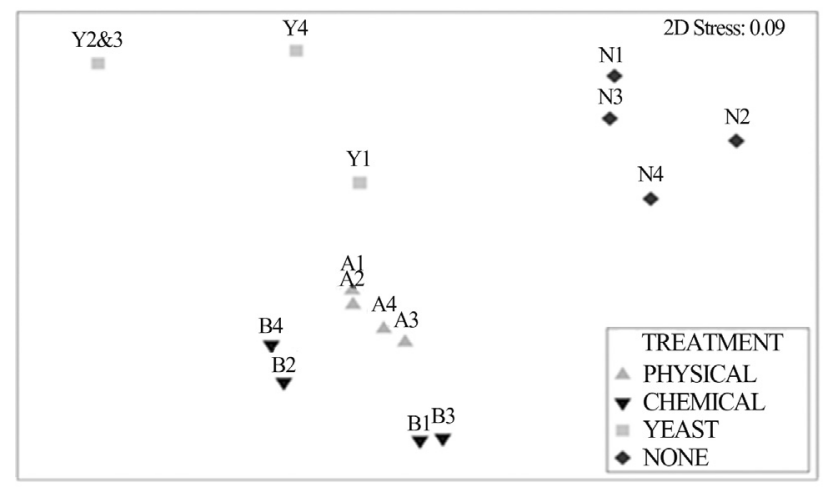

Figure 3 - An NMDS plot of DGGE bands, showing the clustering of replicate samples from different treatments.
The components of the chemical treatment (Treatment B) were expected to destroy viable cells on the leaf surface (Arnold et al., 2007), yet the PCR amplification of $S$. cerevisiae DNA sequences (Figure 1) from surface treated leaves demonstrated that epiphytic yeast DNA sequences were not eliminated, as predicted by Guo (2010). PCR is more sensitive in detecting fungi than traditional culture plating (Baek and Kenerley, 1998), which emphasizes the importance of recognizing that microbial sterility does not guarantee the elimination of extraneous or epiphytic DNA.

ANOVA analysis (Table 1) of the agarose gel (Figure 1) confirmed that Treatments A and B did not eliminate the yeast DNA, but significantly reduced its presence relative to the positive control (Y). The lower $\mathrm{CV} \%$ value in Treatment A indicated that this was the more consistent method of the two.

The ideal outcome of any surface treatment revealed by DGGE gel data would be no amplification of the yeast or epiphytic DNA with as many other bands as possible, while bearing minimal similarity in DNA band composition to the control samples. In the contour map (Figure 2) Region P was largely populated by the yeast amplicons, which were strongly represented in the positive control (Y), due to the high presence of yeast DNA. The relative absence of 


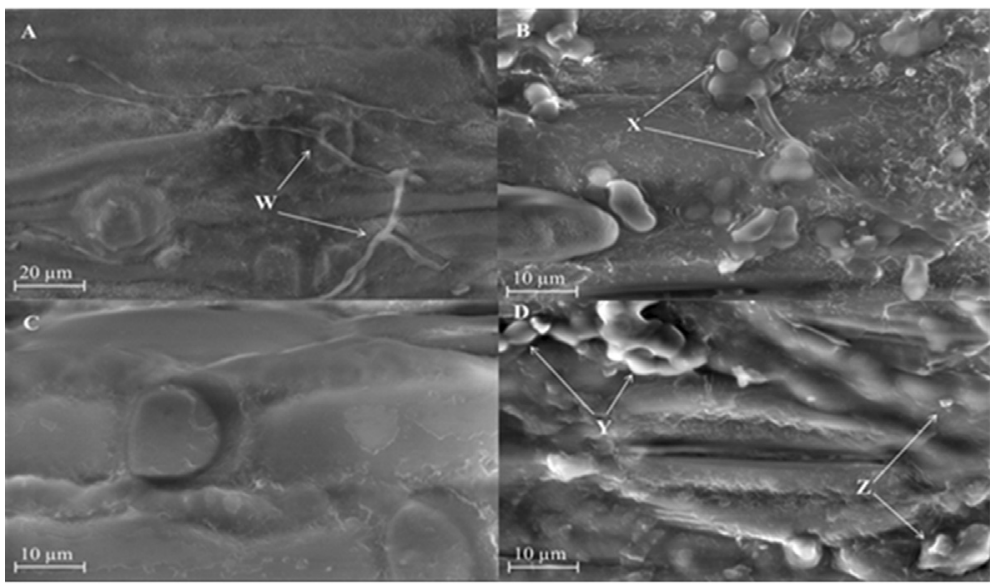

Figure 4 - VPSEM micrographs of A-an uninoculated and untreated leaf surface (Negative Control-N) showing fungal hyphae (W), B-inoculated and untreated leaf surfaces (Positive Control-Y) showing abundant yeast cells (X), C-inoculated and physically abraded leaf surfaces (Treatment A) indicating a significant reduction in debris and $S$. cerevisiae cells, and D-inoculated and chemically surface sterilized leaf surfaces (Treatment B) indicating the presence of $S$. cerevisiae cells (y) and debris (z).

amplicons in Region Q and increased band density in Region $\mathrm{R}$ for the two treatments ( $\mathrm{A}$ and $\mathrm{B}$ ), compared to the negative control $(\mathrm{N})$, suggested that both surface treatments resulted in an enhanced amplification of endophyte target sequences due to reduced competition for primers (von Wintzingerode et al., 1997) by epiphytic target sequences.

The PERMANOVA analysis implied that the treatments varied in their effect on epiphyte diversity. The mean similarity percentages showed that Treatment A produced the most consistent band profiles (Table 2), arguing in its favor as the preferred treatment of the two.

The NMDS plot (Figure 3) exhibited compromised consistency of the chemical treatment (B); however it was less similar to the two controls ( $\mathrm{Y}$ and $\mathrm{N}$ ) than the physical treatment (A). Because of this it was speculated that the chemical treatment damaged target sequences belonging to endophytes as well, even though there was no significant difference in mean band numbers for the two treatments.

VPSEM images (Figure 4) showed qualitative differences between the treatments. The abundance of $S$. cerevisiae cells on the inoculated but untreated control (Figure 4B) correlated with the pronounced PCR amplification of S. cerevisiae (Figure 1). The VPSEM showed that physical abrasion (Figure 4C) was more efficient in removing microbes and debris than chemical treatment (Figure 4D), although the PCR analysis revealed no significant difference (Table 1), therefore VPSEM results alone may not provide a reliable evaluation method in DNA-based studies.

From this data, we would recommend that the physical abrasion technique is superior to the chemical technique along the criteria of greater consistency. This higher-input method may perform better, but larger sample sizes would favor the ease and rapidity of the chemical treatment, which produced the same number of bands found in physically abraded leaves, even though profiles differed slightly.
Since the initial analysis from the agarose gel (Figure 1 and Table 1) was supported by the subsequent analyses of DGGE gel data (Figures 2 and 3; Table 2), we propose that this procedure alone constitutes adequate investigative effort when striving to optimize surface DNA removal techniques in DNA-based fungal endophyte studies, using $S$. cerevisiae as a control organism.

\section{Acknowledgments}

This material is based upon work supported financially, in part, by the National Research Foundation of South Africa and the Winter Cereal Trust. All climate data was courtesy of the Agrometeorology Discipline, School of Agricultural, Earth and Envrionmental Sciences, University of KwaZulu-Natal, Pietermaritzburg, South Africa.

\section{References}

Anand R, Paul L, Chanway C (2006) Research on endophytic bacteria: recent advances with forest trees. In: Schulz, B., Boyle, C., Sieber, T.(eds). Microbial Root Endophytes, Vol. 9. Springer-Verlag, Berlin, p. 89-103.

Anderson MJ (2001) A new method for non-parametric multivariate analysis of variance. Austral Ecol 26:32-46.

Arnold AE, Henk DA, Eells RL, Lutzoni F, Vilgalys R (2007) Diversity and phylogenetic affinities of foliar fungal endophytes in loblolly pine inferred by culturing and environmental PCR. Mycologia 99:185-206.

Backman PA, Sikora RA (2008) Endophytes: An emerging tool for biological control. Biol Cont 46:1-3.

Baek JM, Kenerley CM (1998) Detection and enumeration of a genetically modified fungus in soil environments by quantitative competitive polymerase chain reaction. FEMS Microbiol Ecol 25:419-428.

Clarke, KR, Gorley, RN (2006) PRIMER v6: User Manual/Tutorial. PRIMER-E, Plymouth. 
Crous PW, Petrini O, Marais GF, Pretorius ZA, Rehder F (1995). Occurrence of fungal endophytes in cultivars of Triticum aestivum in South Africa. Mycoscience 36:105-111.

Guo LD (2010) Molecular diversity and identification of endophytic fungi. In: Gherbawy, Y., Voigt, K.(eds). Molecular Identification of Fungi. Springer, Berlin, p. 277-296.

Kemp BM, Smith DG (2005) Use of bleach to eliminate contaminating DNA from the surface of bones and teeth. Forensic Sci Int 154:53-61.

Larran S, Perelló A, Simon MR, Moreno V (2002) Isolation and analysis of endophytic microorganisms in wheat (Triticum aestivum L.) leaves. World J Microbiol Biotechnol 18:683-686.

Lindow SE, Brandl MT (2003) Microbiology of the phyllosphere. Appl Environ Microbiol 69:1875-1883.

Manter D, Delgado J, Holm D, Stong R (2010) Pyrosequencing reveals a highly diverse and cultivar-specific bacterial endophyte community in potato roots. Microbial Ecol 60:157166.

McCune B, Grace JB, Urban DL (2002) Analysis of ecological communities (Vol. 28). MjM software design, Gleneden Beach, Oregon.

Meyer JM, Hoy MA (2008) Removal of fungal contaminants and their DNA from the surface of Diaphorina citri (Hemiptera: Psyllidae) prior to a molecular survey of endosymbionts. Fla Entomol 91:702-705.

Müller C, Riederer M (2005) Plant surface properties in chemical ecology. J Chem Ecol 31:2621-2651.

Payne RW, Murray DA, Harding SA, Baird DB, Soutar DM (2011) An Introduction to Genstat for Windows (14th Edition). VSN International, Hemel Hempstead, UK.

R Core Team (2013). R: A language and environment for statistical computing. R Foundation for Statistical Computing, Vi- enna, Austria. Available at http://www.R-project.org. Accessed 24 November 2013.

Schulz B, Wanke U, Draeger S, Aust HJ (1993) Endophytes from herbaceous plants and shrubs: effectiveness of surface sterilization methods. Mycol Res 97:1447-1450.

Sessitsch A, Reiter B, Pfeifer U, Wilhelm E (2002) Cultivation-independent population analysis of bacterial endophytes in three potato varieties based on eubacterial and Actinomycetes-specific PCR of 16S rRNA genes. FEMS Microbiol Ecol 39:23-32.

Stokes D (2008) Principles and Practice of Variable Pressure/Environmental Scanning Electron Microscopy (VP-ESEM). John Wiley and Sons, Ltd, Chichester.

Tottman DR (1987) The decimal code for the growth stages of cereals, with illustrations. Ann Appl Biol 110:441-454.

von Wintzingerode F, Göbel UB, Stackebrandt E (1997) Determination of microbial diversity in environmental samples: pitfalls of PCR-based rRNA analysis. FEMS Microbiol Rev 21:213-229.

Wakelin SA, Colloff MJ, Harvey PR, Marschner P, Gregg AL, Rogers SL (2007) The effects of stubble retention and nitrogen application on soil microbial community structure and functional gene abundance under irrigated maize. FEMS Microbiol Ecol 59:661-670.

Warnes GR, Bolker B, Bonebakker L, Gentleman R, Huber W, Liaw A, Lumley T, Maechler M, Magnusson A, Moeller S, Schwartz M, Venables B (2013) gplots: Various R programming tools for plotting data. Rpackage version 2.12.1. http://www.cran.r-project.org/web/packages/gplots.

All the content of the journal, except where otherwise noted, is licensed under a Creative Commons License CC BY-NC. 